Please do not remove this page

RMIT

UNIVERSITY

\title{
Efficient frequent pattern mining on web logs
}

Sun, Liping; Zhang, Xiuzhen

https://researchrepository.rmit.edu.au/esploro/outputs/9921864009301341/filesAndLinks?institution=61RMIT_INST\&index=null

Sun, L., \& Zhang, X. (2004). Efficient frequent pattern mining on web logs. Advanced Web Technologies and Applications: Sixth Asia-Pacific Web Conference, APWeb 2004, 533-542.

https://doi.org/10.1007/b96838

Published Version: https://doi.org/10.1007/b96838

Repository homepage: https://researchrepository.rmit.edu.au

(c) Springer-Verlag Berlin Heidelberg 2004

Downloaded On 2023/04/26 13:41:51 +1000

Please do not remove this page 


\title{
Efficient Frequent Pattern Mining on Web Log Data
}

\author{
Liping Sun ${ }^{*} \quad$ Xiuzhen Zhang \\ RMIT University RMIT University
}

\section{Paper ID: 304}

\begin{abstract}
Mining frequent patterns from web log data can help to optimise the structure of a web site and improve the performance of web servers. Web users can also benefit from these frequent patterns. Many efforts have been done to mine frequent patterns efficiently. Candidate-generation-and-test approach (Apriori and its variants) and pattern-growth approach (FP-growth and its variants) are the two representative frequent pattern mining approaches. Neither candidate-generation-and-test approach nor pattern-growth approach is always good on web log data. We have conducted extensive experiments on real world web log data to analyse the characteristics of web logs and the behaviours of these two approaches on web log data. We propose a new algorithm - Combined Frequent Pattern Mining (CFPM) algorithm to cater for web log data specifically. We use some heuristics in web log data to prune search space and reduce many unnecessary operations in mining, so that better efficiency is achieved. Experimental results show that CFPM can significantly improve the performance of pattern-growth approach by $1.2^{\sim} 7.8$ times on frequent pattern mining on web log data.
\end{abstract}

${ }^{*}$ Contact author: Ms Liping Sun, School of CS \& IT, RMIT University GPO Box 2476v, Melbourne 3001, Australia. Email: lisun@cs.rmit.edu.au

Citation:

Sun, L and Zhang, X 2004, 'Efficient frequent pattern mining on web logs', in JX Yu et al. (ed.) Advanced Web Technologies and Applications: Sixth

Asia-Pacific Web Conference, APWeb 2004, Berlin, 15 March 2004. 


\section{Introduction}

The web can be viewed as the largest database available and is a challenging task for data mining. By discovering and analysing the web data, we can save more work time and get more useful information. Web mining is a combination of the data mining technology and the web technology. Web mining includes web content mining, web structure mining and web usage mining [9]. With web usage mining, we extract and analyse useful information such as traversal patterns of users from web log data. Both web designers and web users benefi t from web usage mining. On one hand, by analysing the traversal patterns in web server's log, the designers of web site can determine web users' browsing behaviours so they can know which is the most popular page on a site and which pages are likely visited together. On the other hand, users of the web can also take advantage of this to access the web more effi ciently. Mining frequent traversal patterns is one of the most important techniques in web usage mining.

Web logs are the source data of web usage mining. Usually a web server registers in each web log entry the IP address from which the request originated, the URL requested, the date and time of accesses, the page references and the size of request data. To analyse web $\operatorname{logs}$, the fi rst stage is to divide web log records into sessions, where a session is a set of page references of one source site during one logical period. Practically a session can be seen as a user starting visiting a web site, performing work, and then leaving the web sites.

The most common data mining technique used in web usage mining is that of uncovering traversal patterns. Based on whether web pages in a pattern are ordered or not, duplication is allowed or not, consecutive or not. Several different types of traversal patterns have been examined [6]: an episode is a subset of related user clicks in a session, in which web pages are ordered, not duplicate and not consecutive; a sequential pattern is a sequential series of web pages viewed in a session, in which web pages are ordered, no necessarily consecutive, duplication is allowed. In this study, we focus on mining frequent patterns from web logs. A frequent pattern is the set of web pages visited together in a session, whose support is above the minimum support threshold. In this regard, repeated visited web pages are ignored and web pages are not necessarily ordered and consecutive. Frequent patterns are very helpful to identify pages accessed together in one session.

Let $P=\left\{p_{1}, p_{2}, \ldots, p_{n}\right\}$ be the complete set of web pages. Let $W$ be the web log to be 
analysed. $W$ is a set of sessions, $W=\left\{S_{1}, S_{2}, \ldots, S_{m}\right\}$ and $S_{i}=\left\{p_{1}, p_{2}, \ldots, p_{i}\right\}$ where $p_{i} \in P, 1 \leq i \leq n$. The set $p=\left\{p_{1}, p_{2}, \ldots, p_{i}\right\}$ is a set of pages or clicks. $p$ is called a pattern. $\operatorname{supp}(p)$ is the support of a pattern and is the percentage of sessions that contain the pattern. $\alpha$ is the predefi ned minimum support threshold. If $\sup p(p) \geq \alpha$ then $p$ is a frequent pattern. As we discussed earlier the traversal patterns can be different regarding order, duplication and consecutiveness, in this paper, we are talking about frequent patterns without duplication and ordering, and the web pages in one pattern are no necessarily consecutive. For example, there is a session $S_{1}=\{a b a c d\}$, after data pre-processing this session become $\{a b c d\}$. Following the terminology in traditional data mining, $P$ is the item space, sessions are transactions and pages are items.

Frequent pattern (FP) mining has been studied extensively in mining supermarket transaction data and relational data. Many FP-mining algorithms have been proposed. The Apriori algorithm [1] is a seminal algorithm for fi nding frequent patterns. The name of the algorithm "Apriori" is based on the fact that the algorithm uses prior knowledge of frequent patterns properties, which means all nonempty subsets of a frequent pattern must also be frequent. This is also known as Apriori heuristic. Apriori adopts the candidate-generationand-test approach to find frequent patterns. The Apriori heuristic is used to generate candidate frequent patterns and prune the search space. With Apriori, database need to be repeatedly scanned to compute FPs. The number of passes of scan is equal to the items of the longest pattern plus 1. FP-growth [3] is another representative frequent pattern mining algorithm. FP-growth adopts a pattern-growth approach and divide-and-conquer strategy in search. A prefi x tree called FP-tree is used to compress and represent the input dataset. During mining stage the tree is divided into a set of subtrees for each frequent item. Mining is conducted on each subtree. Longer patterns are grown from short patterns. Trees conditioned on short frequent patterns are repeatedly constructed and searched to produce longer patterns. AFOPT (Ascending Frequency Order Prefi x Tree) [4] is a variant of FPtree. The AFOPT-mining algorithm, which uses the top-down traversal strategy and the ascending frequent item ordering method. Reported experimental results show that the AFOPT-mining algorithm achieves signifi cant performance improvement over FP-growth. Apriori-All and GSP algorithms are proposed in [21] to mine sequential patterns, which are ordered, no duplicate and no necessarily consecutive. In [5], Web Access Path trees (WAP trees) were proposed to mine web access patterns from web logs. Web access patterns are 
sequential patterns, where web pages are ordered, no necessarily consecutive and repetition is allowed. WAP-tree and the WAP-mining algorithm are in essence the FP-tree and the FP-growth algorithm. The main difference between FP-tree and WAP-tree is that ordering is important for WAP-tree.

We notice that most of the previous FP mining algorithms are based on the IBM-artifi cial supermarket transaction data or relational data $[1,3,4,21]$. The dataset of the experiment of WAP-mining is also artifi cial data [5]. In this study, we aim to (1) examine the features of the web log data and frequent patterns presented in the data; (2) compare the performance of the representative FP mining approaches - candidate-generation-and-test approach (Apriori based) and pattern-growth approach (FP-growth based) on mining FPs from web logs; (3) propose better approach more suitable for web logs. Our contribution is as follows: (1) extensive experiments have been conducted to show the difference of the web log data from the artifi cial supermarket transaction data and relational data, in particular, the average and maximal transaction size, the number of frequent items, the number of frequent patterns and the maximal length of frequent patterns are examined; (2) extensive experiments have been conducted to show the performance of Apriori and FP-growth on real world web log data in contrast to artifi cial supermarket transaction data and relational data; (3) a frequent pattern mining algorithm - Combined Frequent Pattern Mining (CFPM) algorithm is proposed. CFPM is more suitable for web log data than FP-growth. The main idea of CFPM is to combine candidate-generation-and-test approach and pattern-growth approach. Besides using a predefi ned minimum support threshold, we use a predefi ned minimum length $(L)$ of FPs. In CFPM, short FPs with length less than or equal to $L$ are mined by candidate-generation-and-test approach, while long FPs with length greater than $L$ are mined by pattern-growth approach. We have implemented the CFPM algorithm. Experimental results show that the CFPM algorithm is about 1.2 7.8 times faster than the pattern-growth approach on mining web log data.

The organisation of the rest of this paper is as follows. Section 2 is the characteristics of web logs and frequent traversal patterns we have observed. Section 3 is mining FPs from web logs, in which we describe the performance behaviour of candidate-generation-andtest approach and pattern-growth approach. In section 4 we propose Combined Frequent Pattern Mining (CFPM) algorithm to get better effi ciency in traversal pattern mining. Section 5 is experimental results and analysis. This paper concludes in Section 6. 


\section{Web Logs and Frequent Patterns in Web Logs}

According the model given in Section 1, data pre-processing is conducted. The web $\log$ data is transformed into a uniform format. The web log data are divided into many sessions. "User sessions" are identifi ed by tracking requests received from an individual user (determined by the IP address) until a period of inactivity indicates that the "session" has ended. The details of data pre-processing are out of scope of this paper, for details please refer to $[9,16,17]$.

In our experiments we use web log datasets: BMS-WebView-1 and BMS-WebView-2, downloaded from the KDD-Cup 2000 home page (www . ecn • purdue . edu/KDDCUP). They have been used in the KDD-Cup 2000 competition. They contain several-months of click stream data from two E-commerce web sites. Each item is a product detail view. Each transaction is a web session consisting of all the product detail pages viewed in that session.

To fi nd the specifi c properties of real world web logs, we also have selected relational data and IBM artifi cial supermarket transaction data, which are popularly used in pattern mining fi eld. Mushroom is a classifi cation dataset down-loaded from the UCI machine learning repository (www.ics.uci.edu/ mlearn/MLRepository.html). It contains attributes about edible and poisonous mushrooms. IBM-artificial is the supermarket transaction dataset (T10I4D100K) generated by the data generator from IBM quest project. The URL is (www.almaden.ibm.com/cs/quest/syndata.html). Table 1 describes the characteristics of the datasets we used in our experiments in terms of the size of the data, number of transactions, total occurrences of items, number of distinct items, maximum transaction size, and average transaction size. According to Table 1, we notice that web log data mostly have a very large number of items, and web logs are very large in size. However, the occurrence probability for each item is mostly very low, or only certain items have high occurrence probability, while for most items occurrence probability is very low. The average transaction size is relatively small, so web log data are mostly very sparse in density.

To investigate the different features of FPs in web logs, extensive experiments are done on real-world web logs. We compare these features of web logs with those of the Mushroom and IBM-artificial datasets. We use web log data of BMS-WebView-1 and BMS-WebView-2 as test datasets for our experiments. All these data are real world data. We notice that many 


\begin{tabular}{c|c|c|c|c|c|c}
\hline \multicolumn{1}{c|}{ Dataset } & Size & $\begin{array}{c}\text { Number of } \\
\text { Transactions }\end{array}$ & $\begin{array}{c}\text { Total } \\
\text { Occurrences } \\
\text { of Items }\end{array}$ & $\begin{array}{c}\text { Number of } \\
\text { Distinct } \\
\text { Items }\end{array}$ & $\begin{array}{c}\text { Maximum } \\
\text { Transaction } \\
\text { Size }\end{array}$ & $\begin{array}{c}\text { Average } \\
\text { Transaction } \\
\text { Size }\end{array}$ \\
\hline BMS-WebView-1 & $2.07 \mathrm{M}$ & 59,602 & 149,639 & 497 & 267 & 2.51 \\
BMS-WebView-2 & $5.01 \mathrm{M}$ & 77,512 & 358,278 & 3,308 & 161 & 4.62 \\
Mushroom & $0.7 \mathrm{M}$ & 8,124 & 186,852 & 127 & 23 & 23 \\
IBM-artifi cial & $33 \mathrm{M}$ & 98,413 & 999,926 & 23,423 & 29 & 10 \\
\hline
\end{tabular}

Table 1: Characteristics of datasets

previously published experimental results are based on the artifi cial datasets, such as [5]. We believe that our experiments are more persuasive on the real world datasets, especially for web log data.

A fi gure showing the dataset features: frequency/transaction size is given in Figure 1. We can see that with the two web logs, most transactions are relatively short-with transaction size less than 5. Both BMS-WebView-1 and BMS-WebView-2 have a very small percentage (less than 10\%) of transactions with size up to 30 items. From Table 1 we also know that such web logs usually have a small average transaction size (2.51 and 4.62), which is much smaller than that of Mushroom (23) and IBM-artificial (10). Obviously web logs are sparse data. On the contrary, the Mushroom dataset is dense data. Each transaction contains 23 items. For the IBM-artificial dataset, short and long transactions are well represented, in terms percentage distribution, transactions of length [3-5], [6-10] and [11-30] are around $16 \%, 43 \%$ and $41 \%$ respectively. The maximum transaction size of Mushroom and IBMartificial are 23 and 29 respectively, while those $B M S$-WebView-1 and BMS-WebView-2 are 267 and 161. This means for web logs, although most of transactions are short, there does exist long transactions, which suggests that the average length of FPs is short while there does exist long FPs.

Figure 2 shows the number of frequent items at different support thresholds on these datasets. We can see that on all datasets with the support threshold decreasing the number of frequent items increases, this is especially so on $B M S$-WebView-2 and IBM-artificial. This means that the search space for FPs tends to grow larger at low support thresholds.From Figure 2 we also know that such Web logs usually have a large number (500-3000) of frequent items during mining, while the Mushroom dataset usually produce 150-300 frequent items during mining. The IBM-artificial dataset is in between the two extremes. 


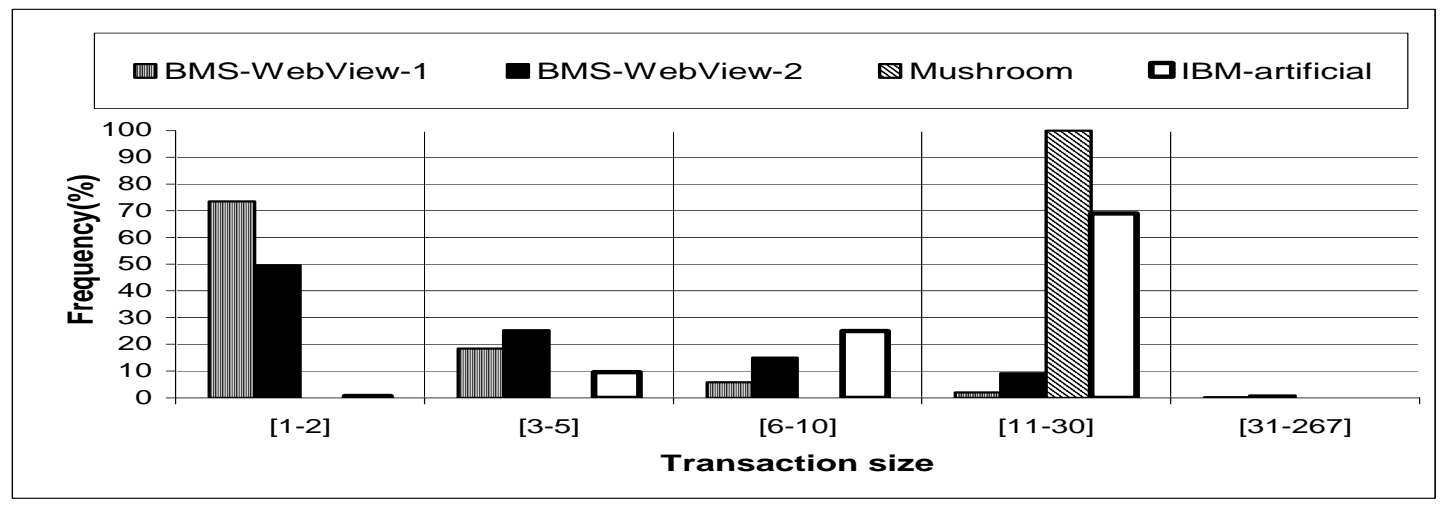

Figure 1: Characteristics of transaction size

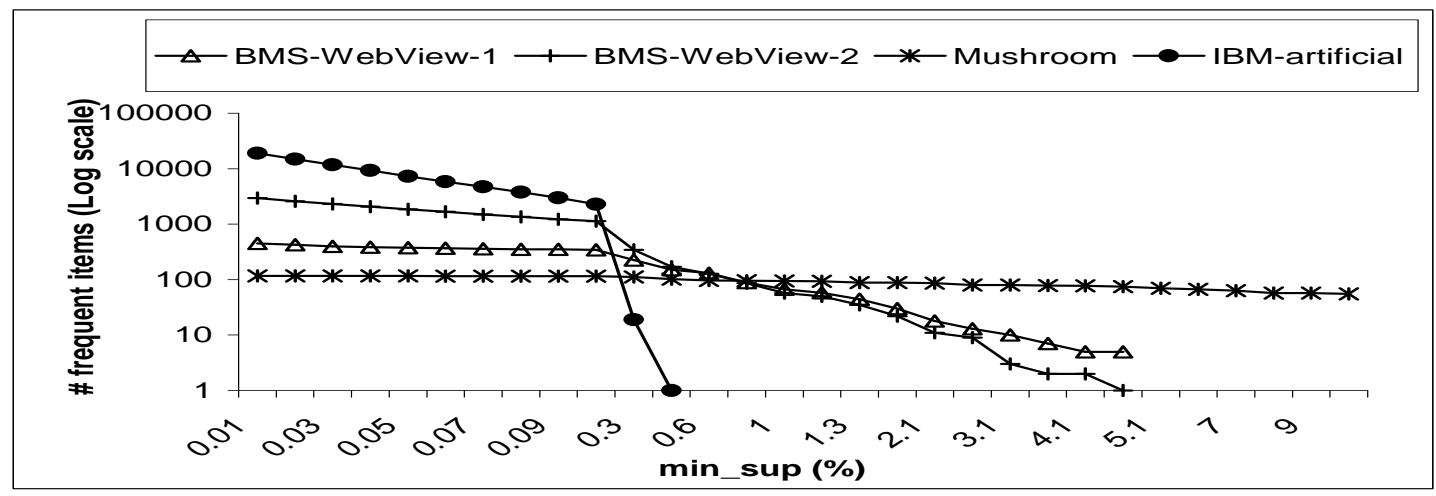

Figure 2: Characteristics of frequent items
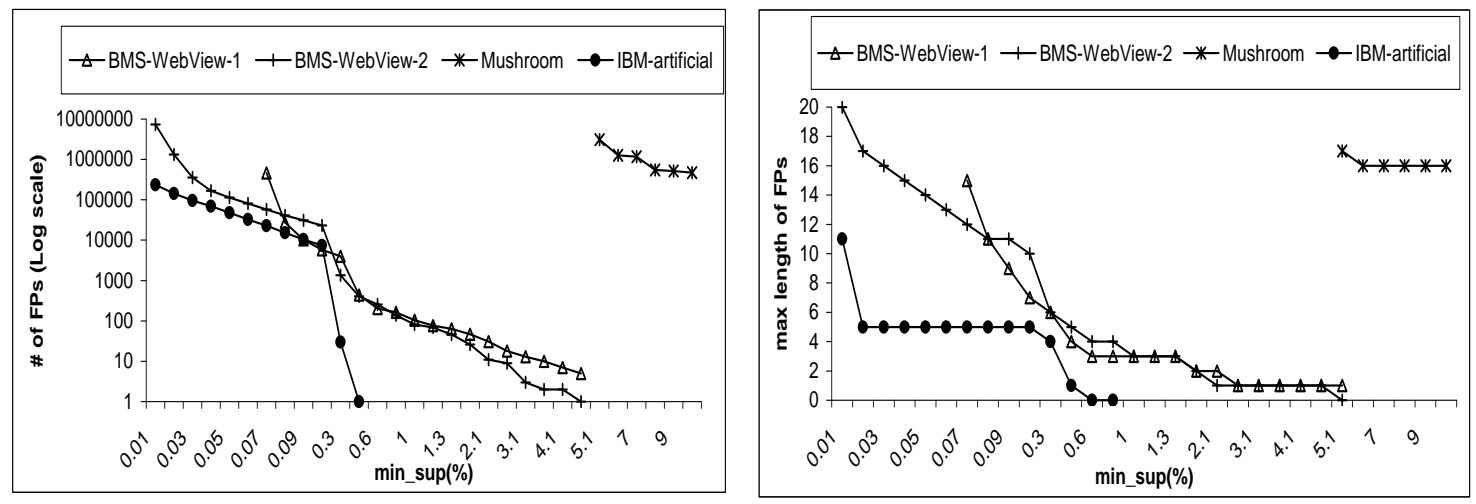

Figure 3: Characteristics of number of FPs

Figure 4: Characteristics of length of FPs 
Figure 3 and Figure 4 show the number of FPs and length characteristics of FPs at different support thresholds on different datasets. The number of FPs increases slow when lowering the threshold. It is obvious from Figure 3 that in general the number of patterns grows at low support thresholds. However, different datasets present different features. On Mushroom there are over 3,000,000 FPs at the support threshold of 5\%. Following the trend of the Mushroom line, we can see that there will be an exorbitant large number of FPs and this is why our experiments stop at 5\%. Most web logs produce less than one million FPs even at low threshold such as $0.01 \%$. An exception is $B M S$-WebView-2, which produces more than $7,000,000$ FPs at threshold $0.01 \%$. From Figure 4 we can see that the maximal length of FPs for BMS-WebView-2 can be 20 when minimal support threshold is about $0.01 \%$. For $B M S$-WebView-1, the curve of the maximal length of FPs has a trend to increase to a large number with the decreasing of minimal support threshold. This is also a reason for that both Apriori and FP-growth cannot complete the mining task, which is shown in Section 3. For IBM-artificial, the length of FPs also has trend to grow longer. However, the trend is not as sharp as that of BMS-WebView datasets. For Mushroom, the length of FPs are always relative longer than others.

In summary, web logs are large in size and sparse in density. The average length of FPs is relative shorter but there does exist long FPs. This characteristics cannot be catered by either Apriori or FP-growth, which we will discussed in details in Section 3.

\section{Mining FPs from Web Logs}

The problem we will solve is to mine frequent patterns in web logs. As web logs are often very large in size but sparse in density, the effi ciency of frequent pattern mining algorithm is important. To investigate the different performance of Apriori and FP-growth in mining FPs on web logs compared that on Mushroom and IBM-artificial, extensive experiments are done on real world web logs. The Apriori implementation we used was down-loaded from the web page fuzzy.cs.uni-magdeburg. de/borgelt/ and was implemented by Christian Borgelt in C. The FP-growth implementation we used was down-loaded from the web page www.cs.helsinki.fi/u/goethals/software/ and the code was implemented by Bart Goethals in C++. All experiments were conducted on a Sun Ultra 80 machine in the School of Computer Science and Information Technology RMIT University. 


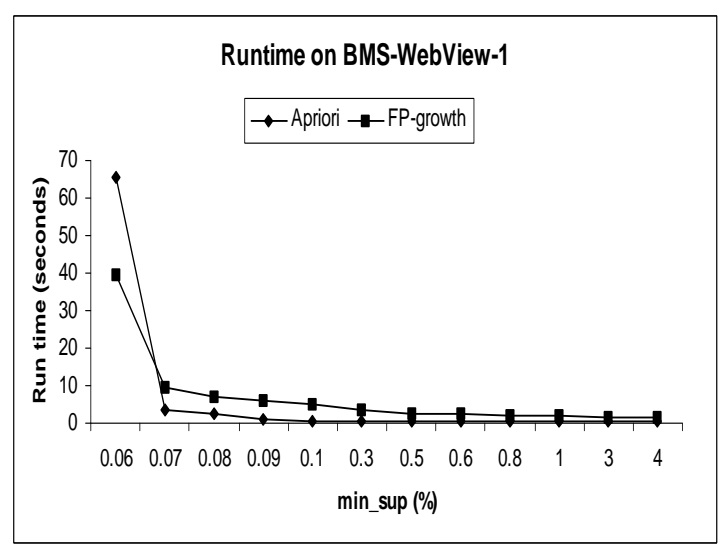

(a)

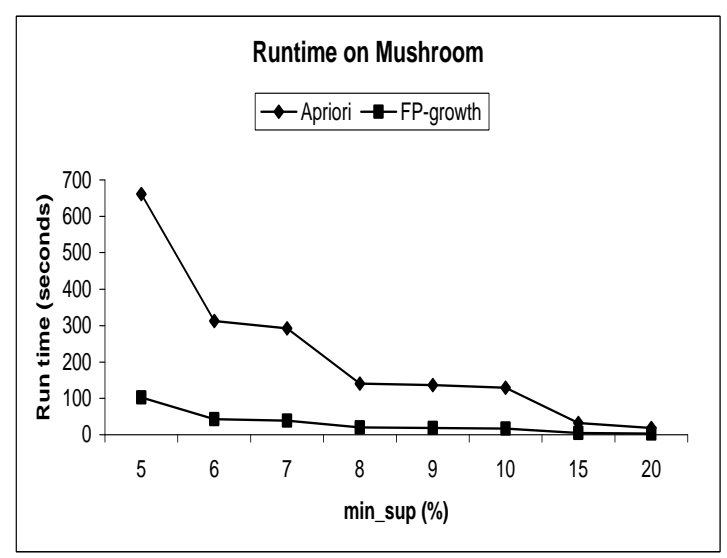

(c)

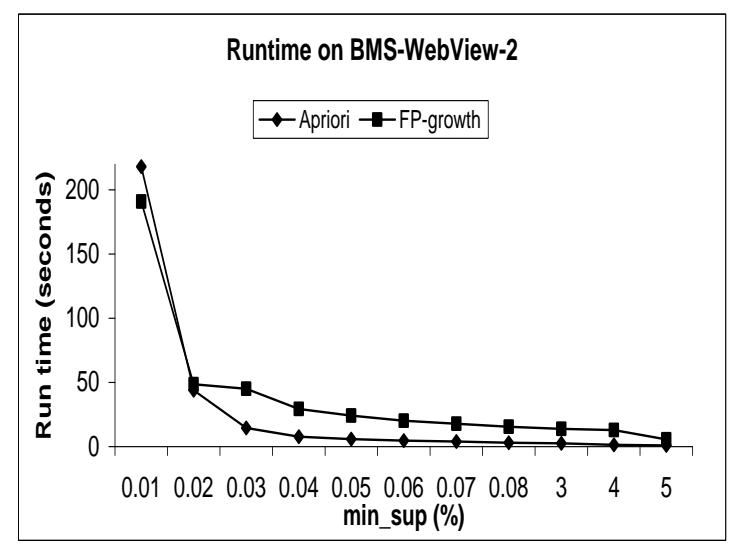

(b)

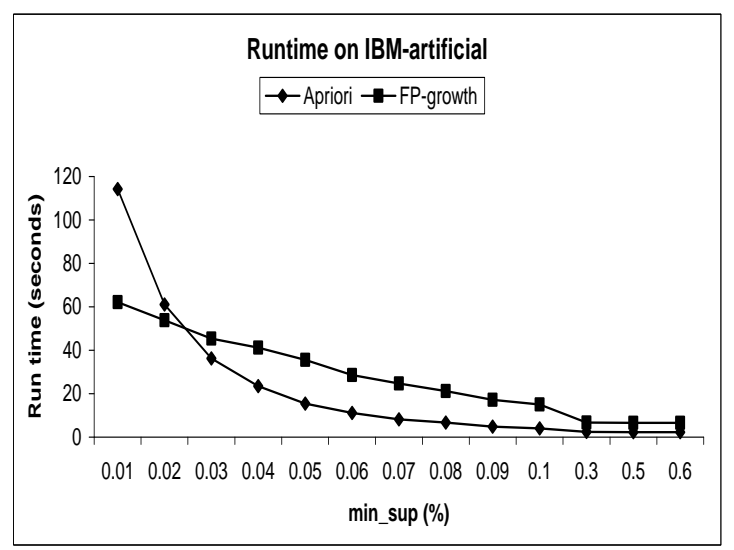

(d)

Figure 5: Performance of Apriori and FP-growth

The system has a main memory of 4.0 GB. and the CPU Speed is $450 \mathrm{MHz}$. The operating system is SunOS 5.8. Timing includes reading, computation and outputting all FPs to disk. The time performance of Apriori and FP-growth are shown in Figure 5. We can see that FP-growth is always better than Apriori on Mushroom, which is a dense datasets and the FPs from it are relative longer with length than 16 (refer to Figure 4). On IBM-artificial when minimal support threshold is higher than $0.02 \%$, the FPs are shorter with length less than 5 and Apriori has better performance. When minimal support threshold is lower than $0.02 \%$, the FPs are longer with length more than 5 and can be 11 and FP-growth has better performance. From Figure 5, we can see on BMS-WebView-1, when minimal support 
threshold is lower than $0.06 \%$, Apriori failed due to too much memory and time needed in candidate generation. FP-growth cannot fi nish mining either. As a result there is no statistics shown when minimal support threshold is lower than $0.06 \%$. However, we also notice that when minimal support threshold is higher than $0.07 \%$, Apriori is better than FP-growth. When minimal support threshold is as low as $0.07 \%$, FP-growth is better than Apriori. At this point, the length of FPs is longer than 12. Similar results are shown on $B M S$-WebView-2. When minimum support threshold is higher than $0.02 \%$, Apriori is better and with the decreasing of the minimum support threshold, the FPs become longer and FPgrowth performs better than Apriori. Especially, BMS-WebView-2 in fact produce a large number of FPs and some of them are indeed long patterns consisting of 20 items. This observation motivates us to propose a new algorithm having better effi ciency on frequent pattern mining on such web log data.

To sum up, we have observed that FP-growth is good at mining dense data with a large number of long FPs. Its performance on web logs is not excellent. On the other hand, Apriori is superior to FP-growth when FPs are relatively shorter. The performance of Apriori is not perfect on web logs either due to its candidate-generation-and-test strategy, which needs big memory space and repeated scanning on the database, so it is resource-devastating in nature. The question is how to improve the existing approaches and find a new approach more suitable for frequent pattern mining on web logs. As will be discussed in Section 4, our solution is to combine the candidate-generation-and-test approach and the pattern-growth approach. We take the advantages of both approaches to avoid expensive candidate-generation-and-test and reduce the cost in pattern-growth.

\section{Combined Frequent Pattern Mining (CFPM)}

In this section, we will answer the question of how to fi nd a more effi cient approach for frequent pattern mining on web logs. We propose a new frequent pattern mining algorithm - Combined Frequent Pattern Mining (CFPM). The major cost of Apriori is too much scanning on database and too many of candidate sets generated. The major cost of FPgrowth is that conditional FP-trees have to be recursively constructed and searched. To further improve the performance of FP-growth, we will discuss how to improve the tree structure and also the way that the tree is searched. 
From previous analysis we have examined when FPs are relatively short, Apriori is superior to FP-growth, while for long FPs, FP-growth is superior. We have analysed the characteristics of FPs mined from web logs. We notice that most of FPs mined from web $\operatorname{logs}$ are short while there do exist some longer patterns, such as patterns with length 20 for $B M S$-WebView-2. Based on these observations, we are trying to combine the advantages of Apriori and FP-growth to gain a better effi ciency of frequent pattern mining on web logs.

It has been recognised and our experiments have also confi rmed that the candidate-generationand-test approach is more suitable for sparse datasets with short patterns, whereas the pattern-growth approach is more suitable for dense datasets with long patterns. In designing our algorithm, we aim at avoiding the costly candidate generation-and-test and improving the performance of the pattern growth approach on mining sparse data such as web logs.

Our algorithm is called Combined Frequent Pattern Mining (CFPM), which combines the candidate-generation-and-test approach and the pattern growth approach. We use candidategeneration-and-test approach to get the FPs with length less than or equal to a predefi ned minimum pattern length $L$. After this we build an Improved Frequent Pattern Tree (IFPtree), which will be discussed in details in Section 4.1. The patterns longer than $L$ are mined from the IFP-tree by frequent pattern growth approach. In fact we use the mining strategy of AFOPT [4], which applies a "frequency ascending order prefi $\mathrm{x}$ tree and top-down traversal" strategy and will be discussed in details in Section 4.3.

\subsection{Improved Frequent Pattern Tree}

In this section we fi rst analyse the merits and weakness of FP-tree [3], which is the underlying data structure of pattern-growth approach. Based on this, we propose an Improved Frequent Pattern Tree (IFP-tree), which is used in CFPM approach. IFP-tree is also based on the FP-tree [3].

The FP-tree was proposed in [3] is the base data structure of pattern-growth approach. A FP-tree is a prefi $x$ tree. The tree is defi ned by frequent items in the data and represents all possible FPs in the datasets. In constructing the tree, frequent items are ordered in frequency-descending order. In the FP-growth algorithm, the FP-tree in frequent item descending order for the sample database of Table 2 is shown in Figure 6. Another important 


\begin{tabular}{l|l|l|l}
\hline TID & Transactions & $I$ (frequency-descending order) & $I$ (frequency-ascending order) \\
\hline 1 & a, b, e, f, m, s & a, e, b, f, m, s & s, b, f, m, a, e \\
2 & a, d, g, k, l & a, d & d, a \\
3 & b, d, e, f, m, s, y & e, b, d, f, m, s & s, b, d, f, m, e \\
4 & a, c, e, m, o & a, e, m & m, a, e \\
5 & a, d, t, u & a, d & d, a \\
6 & b, e, f, p & e, b, f & b, f, e \\
\hline
\end{tabular}

min_sup $=2$, Frequent items $I=\{\mathrm{a}: 4, \mathrm{e}: 4 \mathrm{~b}: 3, \mathrm{~d}: 3 \mathrm{f} ; 3, \mathrm{~m}: 3, \mathrm{~s}: 2\}=\{\mathrm{s}: 2, \mathrm{~b}: 3, \mathrm{~d}: 3, \mathrm{f}: 3, \mathrm{~m}: 3, \mathrm{a}: 4, \mathrm{e}: 4\}$

Table 2: A sample database $D$

concept for a prefi $\mathrm{x}$ tree in pattern-growth approach is the conditional tree for a frequent item. With the FP-tree in Figure 6, the conditional tree for $s$ consists of the two branches, which are the two paths from the root to leaf nodes containing $s$ in Figure 6. To fi nd patterns containing $s$ only these two conditional trees need to be considered.

As shown in the experimental results in Section 3, FP-growth is faster than Apriori when mining long FPs. Reported experimental results show AFOPT-mining [4] is faster than FP-growth. Both FP-growth and AFOPT-mining adopt a pattern-growth approach to mine FPs. Pattern-growth is achieved by the concatenation of the suffi x pattern with the frequent items generated from a conditional FP-tree. Mining starts with frequent 1-item patterns (as the initial suffi $x$ patterns), which are at the bottom of the FP-tree. In FP-growth mining starts from a length-1 FP. Its conditional pattern bases are examined and its (conditional) FP-trees are constructed. Mining is performed recursively with this tree.

To speed up search on FP-tree, in FP-growth [3] a header table consisting of node links is also created, where each node link can be seen as an index to nodes containing a certain item. Especially during the mining, the header table is essential for traversing all paths with the same item. The method of using least frequent items as a suffi x offers good selectivity and substantially reduces the search costs [3], however the cost is still enormous. In FPgrowth, the traversal of the FP-tree is from bottom to top along node-links. Each time the mining starts with visiting the header table to fi nd an item as a conditional base and after that following the node-links to fi nd all nodes in the FP-tree with the same item, then growing the pattern bottom up (the traversal direction is from leaf to root). As a result FP-growth needs to traverse as many branches as the number of leaf nodes in the FP-tree and each node is visited as many times as its total number of descendants. 


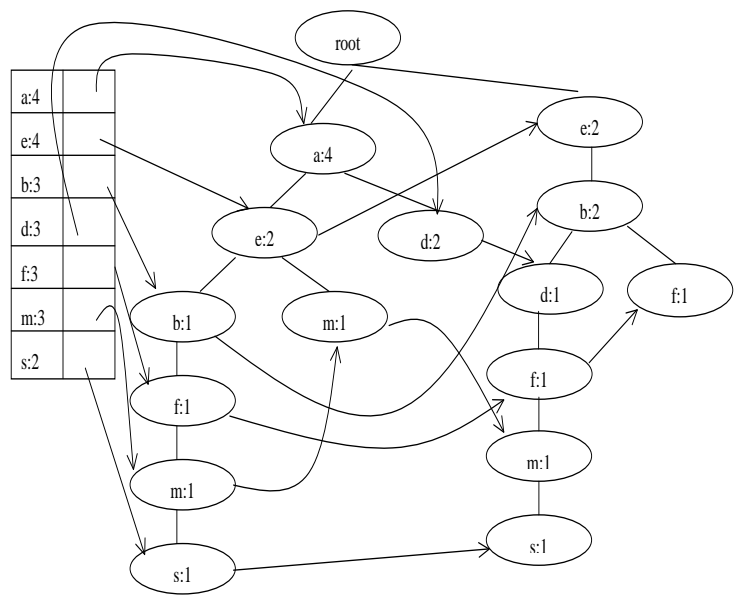

Figure 6: The FP-tree for $D$

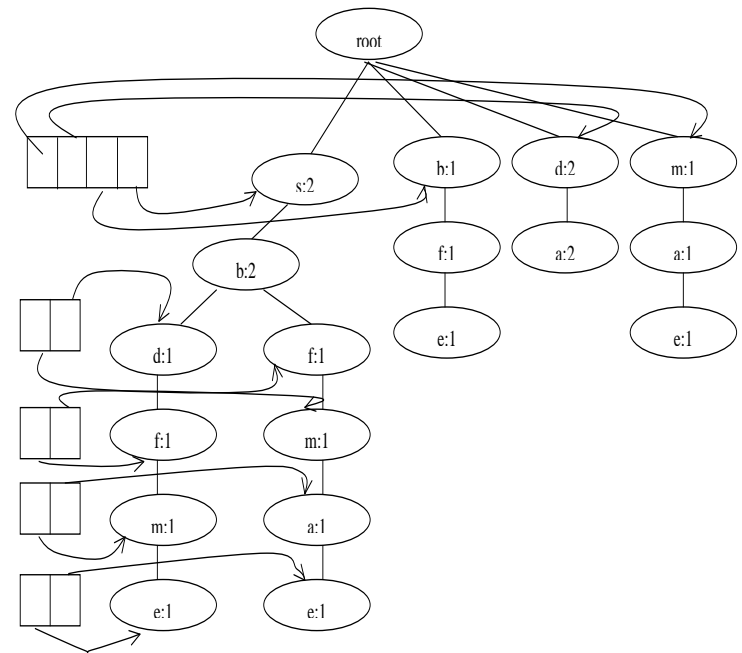

Figure 7: The IFP-tree for $D$

In [4], the AFOPT-mining algorithm uses the Ascending Frequency Order Prefi $\mathrm{x}$ Tree (AFOPT) and top-down traversal strategy for mining FPs, which is capable of minimising the traversal cost of conditional trees. With frequent ascending order, the number of node visits equals the size of the AFOPT tree, which is usually smaller than the total length of tree branches that FP-growth needs to traverse. Similar to FP-growth, during mining it repeatedly constructs header tables and build conditional trees to mine all FPs.

FP-growth uses the frequency descending order. The tree nodes in an FP-tree are arranged in such a way of sharing: more frequently occurring nodes have better chances of sharing nodes than less frequently occurring ones. This approach can minimise the original FPtree, but in the processing of mining, the number of the conditional databases for each item may becomes very large. In addition the total number of conditional databases also becomes very large. On the contrary, we use the frequency ascending order approach during constructing our proposed IFP-tree. With frequency ascending order, nodes sharing among different transactions in the original database is reduced, but it can dramatically minimise the number of the conditional databases for each item as well as the total number of conditional databases.

We have seen that the traversal cost and the cost for constructing header table and conditional trees are the major overheads of the pattern growth approach. We address both problems in designing our CFPM algorithm. We propose Improved Frequent Pattern tree (IFP-tree) based on these observation and analysis. Figure 7 is the IFP-tree for the sam- 
ple database. There are some differences between the FP-tree and IFP-tree: (1) FP-tree is organised in frequency-descending while IFP-tree is in frequency-ascending order. (2) FP-tree has header table while IFP-tree has no header table but has indices for tree nodes at same level. The properties of IFP-tree make it suitable for our CFPM algorithm. The IFP-tree is in frequency-ascending and contains more single paths than the FP-tree, which is in frequency-descending. Since we have to check a path of the FP-tree is single path or not to make sure the support of patterns in this path is global support or not, which will be discussed in details in Section 4.2. Another thing we notice is that the IFP-tree has more branches at its high level than the FP-tree. More frequent items are near the root of a tree so that it can reduce traversal cost. In addition, we do not need the header table, which is a must in the FP-growth approach, so much space and construction time is saved in building tree. As a result, the process of building the IFP-tree is expedited dramatically. The header table in FP-tree is a must, whereas maintaining the header table is very expensive both in time and memory and it incurs more traversal cost during mining phase. There is no header table in the IFP-tree, whereas in IFP-tree we use an index for the frequency order of all the children nodes of the same parent node. This index can expedite searching and inserting both in building IFP-tree phase and mining phase. To maintain all the children nodes of the same parent node in frequency ascending order is critical to ensure the correctness of the mining process, which will be discussed in detail in Section 4.2.

\subsection{Pushing Right}

We first present some observations about the IFP tree, as they ensure the correctness of our CFPM algorithm.

Lemma 4.1 Given a IFP tree $T$ built according to the ordered list of frequent items $I=$ $\left\{i_{1}, i_{2}, \ldots, i_{m}\right\}$ based on partial order relationship $\preceq$, the leftmost branch of $T$ contains the complete information for FPs containing $i_{1}$ and any items $i \nprec i_{1}$.

Rationale: The correctness of the lemma is ensured from the construction process of an IFP tree. In Figure 7, the left branch of the root of the IFP-tree contains all information about $s$-based FPs.

Given an IFP-tree, the leftmost subtree $T_{i_{1}}$ contains the complete information for FPs containing $i_{1}$, but not the other subtrees. By pushing the information in $T_{i_{1}}$ to its right sibling 
sub-tree, or pushing the sub-trees right to its sibling trees, we would have the next leftmost subtree $T_{i_{2}}$ contain all information for FPs containing $i_{2}$ and items following $i_{2}$ in $I$. At the mining stage, pushing right is the major overheads of top down traversal. We can avoid some unnecessary pushings such as the fi rst $L$ levels of tree can be skipped. If we have got the FPs with length less than or equal to a predefi ned minimum pattern length $L$ before building IFP-tree, we start checking single path from the $L+1$ level of the tree, when this sub-tree has less than $L$ levels (including $L$ ) subtree, we do not need to push this subtree to its right siblings. Another improvement is that if the count of an item already reaches the global support of it, then we have already got all the FPs containing this item. Again we do not need to push this node right. So the principle is trying to reduce operations but still contain complete pattern information. Function PusingRight $\left(T_{s}, T\right)$ merges the children of $T_{s}$ with those of $T$, as shown in Figure 8.

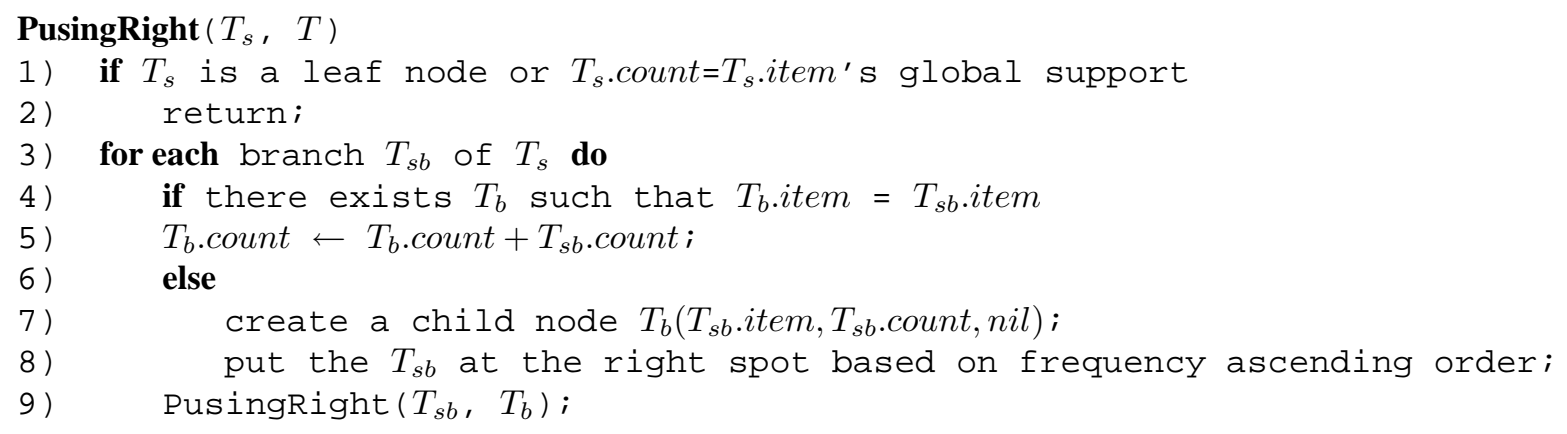

Figure 8: The PusingRight Algorithm

Example 4.2 Figure 9 shows the IFP-tree as the result of pushing right the $s$-subtree of the IFP-tree in Figure 7. We can see now the new leftmost $b$-subtree contains all information needed for mining FPs containing items after $a$ in the item list, which are $b, d, f, m$ and $e$.

Lemma 4.3 Given an IFP-tree, each node $(e: t)$ on the left-most path of the tree represents a pattern the-path-to- $e$ with a global support of $t$.

Rationale: The correctness of Lemma 4.3 is based on the IFP-tree construction process. Each complete set of frequent items in a transaction of the original database is mapped to one path of the IFP-tree. At the tree-building phase, whenever a new transaction is processed, the pointer pointing to the current node of the IFP-tree goes back to the direct child node of the root node. That is, checking always starting from the leftmost branch of the IFP-tree to decide whether a new node is added to the existing IFP-tree or just increase the count of an existing node by 1 . Therefore, each node on the leftmost branch of the 


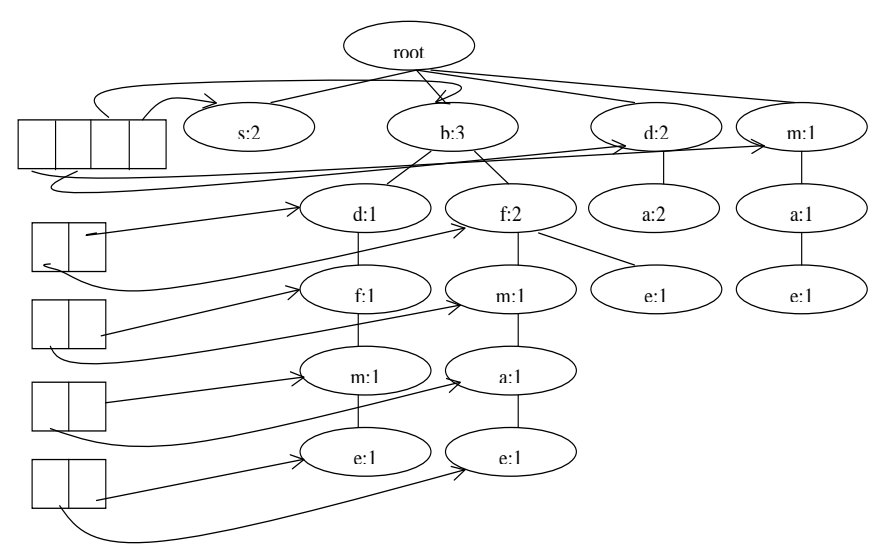

Figure 9: A sample IFP-tree after pushing right

IFP-tree represents a pattern with a global support. Thus we have the Lemma. Notice Line 8 in the PusingRight algorithm in Figure 8, keeping frequency ascending order is critical to ensure the correctness of Lemma 4.3.

Given Lemma 4.3, we can list all frequent patterns from the leftmost branch: the path from root to each node $(e, t)$ with $t \geq \tau$.

Example 4.4 Considering the sample database $D$ of Table 1 and the IFP-tree in Figure 7, with $P=\phi$ we can immediately enumerate all the FPs (with their support) on the leftmost path together with their support, which are $\{\mathrm{s}: 2, \mathrm{sb}: 2\}$. Note however, the non-rooted pattern $b$ does not have the global support.

Definition 4.5 A single-path IFP-tree is an IFP-tree in which all of its descendents are also single-path IFP-trees, which means that none of its descendents has any siblings.

If $T$ is a single-path tree, we can enumerate all frequent patterns conditioned on $P$ combining the nodes with count greater than the threshold.

Example 4.6 Figure 10 gives an example of a single path IFP-tree conditioned on $s b$. Suppose the support threshold is 1 . We can immediately enumerate all the frequent patterns (with their support) that can be inferred from the tree, which are pattern $\alpha \cup$ all the combinations of the nodes in the single path IFP-tree, with checking whether the support of the item of the node is greater than or equal to the support threshold. In this example, the inferred patterns are: $s b d: 1, s b f: 1, s b m: 1, s b e: 1$, sbdf:1, sbdm:1, sbde:1, sbfm:1, sbfe:1, sbme:1, sbfme:1.

It should be noted that Lemma 4.1 and Lemma 4.3 also hold on conditional prefi $\mathrm{x}$ trees. As will be seen later, the operation of "pushing right" guarantees the leftmost branch of the IFP-tree always contains the complete information of FPs. This also guarantees that we can directly output the frequent patterns embedded in the leftmost branch of the IFP-tree with correct global support. This also guarantees the correctness of top down traversal. 


\section{$\{\mathrm{s}, \mathrm{b}\}$}

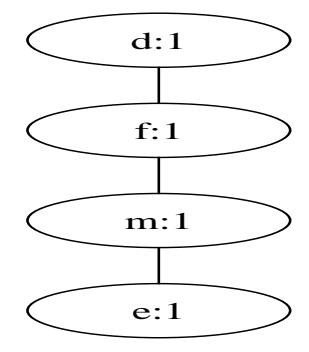

Figure 10: An example of a Single Path IFP-tree

\subsection{Top Down Traversal}

With CFPM, the IFP-tree is traversed top-down to mine FPs by the algorithm Traverse we proposed. Taverse is shown in Figure 11. In Function Traverse, we fi rst check the left-most branch of the IFP-tree is a single path tree or not. If it is a single-path tree, then check the depth of the tree is greater than the predefi ned minimum length of FPs or not. If it is greater than the predefi ned minimum length of FPs, then output the FPs on the path. The patterns are all combinations of the nodes in the single path concatenated with the set of items in the prefi $x$ tree, which is the conditional pattern. The count of the pattern is the count of the conditional pattern. If it is not a single-path tree, we then merge this left-most branch of the IFP-tree, with its siblings. This is the process of "Pushing right". After output patterns in the left-most branch of the IFP-tree and pushing right the pattern information to right siblings, we can safely remove this left-most branch that has been processed to save the memory needed at running time. This process is performed recursively until there is no child node of the global root in the IFP-tree.

Note that this top-down traversal of IFP-tree can be very costly because of some expensive operations. Checking whether a tree is a single path is in fact costly, as we have to recursively check all its subtrees. When there is a single branch remaining and it is a single path tree, we do not need to do pushing to lift all of its descendents as its siblings. Instead we can output all the frequent patterns embedded in it directly.

In growing patterns, the cost of building conditional tree is non-trivial. It is more pronounced when the underlying patterns are short. Addressing this cost of mining short patterns, FPs with their length less than or equal to a predefi ned minimum pattern length $L$ should be mined directly by checking their support. Indeed this can be achieved during the 
Traverse $(T, P, L, \alpha)$

;; Top-down traversal of an IFP-tree for growing frequent patterns.

;; $T$ is the root of the sub-tree under examination,

; $L$ is the minimum length of patterns specifi ed,

;; P passed by reference and the algorithm is started with Traversal( global-FP-tree, $\phi)$,

$; ; \alpha$ is the global support threshold.

;; there only exists the global root node

$1)$ if $T$ is the global root

2) return;

3) else

if $T$ is a single-path tree

if T.depth $>L$ and T.count $>=\alpha$ else output FPs embedded in $\mathrm{T}$ based on the conditinal pattern $P$;

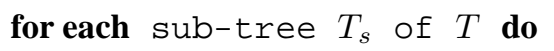

Figure 11: The Traverse Algorithm

tree building stage without any additional scan of data. In building the tree, after the 1st scan on DB, we accumulate the supports for each item and has determined the 1-item FPs and their ordering. During 2nd scan we can also accumulate the supports for 2-item FPs, ..., until $L$-item FPs.

During mining phase we also add some checking conditions: (1) if the count of a node already equal to the global support of the item in it, then this node is no neccesary to be pushed right. The gloabal support for each item has already be accumulated in the reading phase. (2) if a single path tree is less than or equal to $L$, then we do not need to mine this path recursively. The reason is we already get all the frequent patterns with their length less than or equal to a predefi ned minimum pattern length $L$.

In our proposed improvement on FP-growth, we do not need to construct conditional databases as FP-growth does. We can mine patterns directly on the original tree along with some merging operations, which only involve some pointer re-organization and counter increment and a little more new nodes created. Furthermore we remove the branch after it has been processed, which includes outputting patterns embedded in it and pushing information in it to right branches. As a result the total number of nodes does not increase too much and the total running time is much less than FP-growth. In addition, this approach can dramatically save the memory needed in the whole running duration. 


\subsection{The CFPM Algorithm}

Put things together, we have the CFPM algorithm as shown in Figure 12. As analysed earlier, generating short patterns with the pattern growth approach is costly compared to generating long patterns. We thus use the candidate-generation-and-test approach to mine frequent patterns with length less than or equal to a predefi ned minimum pattern length $L$. After that, traversal of IFP-trees starts from the tree nodes of depth $L$.

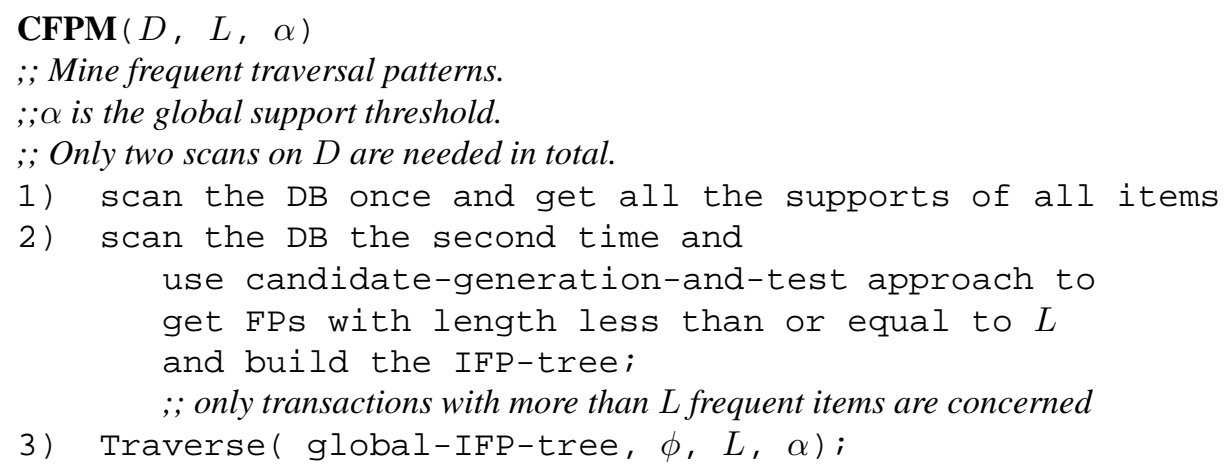

Figure 12: The CFPM Algorithm

Only two scans in total on the database are needed. After the fi rst scan on the database, all the supports of all items are accumulated. Based on the minimum support threshold, all the 1-item FPs are found. In the process of the second scan on the database, based on the 1-item FPs, we use candidate-generation-and-test approach to fi nd 2-item FPs, $\ldots L$-item FPs. At the same time, the IFP-tree is built. If a transaction has less than or equal to $L$ frequent items, then it is pruned so it will not present in the IFP-tree. The long FPs with length more than $L$ will be mined by function Traverse algorithm as shown in Figure 11. The key idea of CFPM is to use candidate-generation-and-test approach to get short patterns, while use pattern-growth approach to mine long FPs.

\section{Experiments}

We have implemented our proposed CFPM algorithm in C++. Experiemts setup are same as discribed in Section 3. Extensive experiments were conducted for the time effi ciency comparison on the two web log datasets we used. In order to show the efficency improved by using the strategy of combining the candidate-generation-and-test approach and the pattern-growth approach, we fi rst conducted experiments on web logs with mining both 


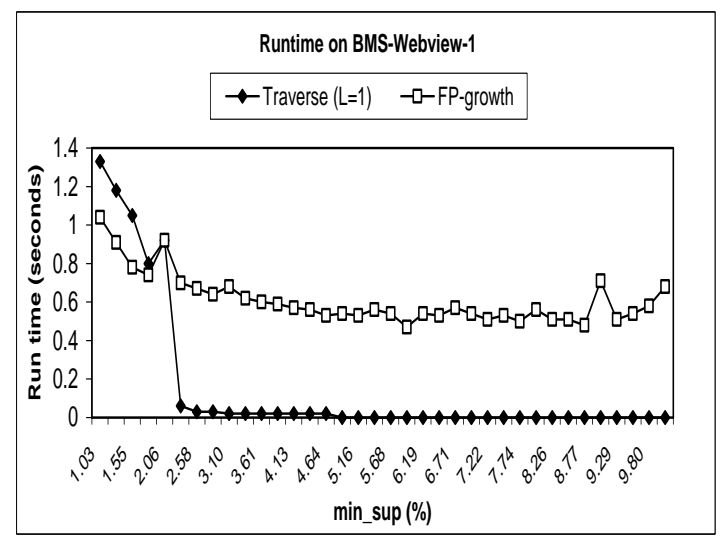

(a)

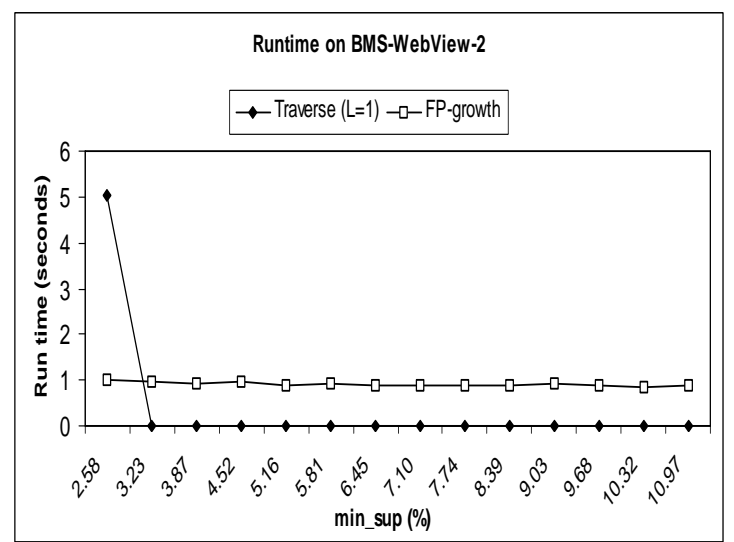

(b)

Figure 13: Performance of Traverse $(L=1)$ and FP-growth

short FPs and long FPs pattern-growth approach, which is in fact Traverse with $L=1$. We have compared the results of Traverse $(L=1)$ and FP-growth. The experimental results are shown in Figure 13.

From Figure 13 we see that on the BMS-WebView datasets, Traverse $(L=1)$ outperforms FP-growth when minimum support threshold is relatively high. Specifi cally, for $B M S$ WebView- 1 when minimum support threshold is greater than $2.5 \%$, Traverse $(L=1)$ is averagely 6 times faster than FP-growth, and for BMS-WebView-2 when minimum support threshold is greater than $3.2 \%$, Traverse $(L=1)$ is also much faster than FP-growth. The reasons are as follows. (1) Without necessary to maintain header table, bulding IFP-tree is much faster than buliding FP-tree. (2) The "Frequency-ascending order + top-down mining" strategy of Traverse $(L=1)$ is superior to "Frequency-desending order + bottom-up mining" strategy of FP-growth. Since the number of conditional databases is much less for the former one.

The experimental results show that only use frequent pattern growth approach (Traverse with $L=1$ ) does not have good effi ciency when the minimum support threshold is relatively low. With the dropping of minimum support threshold, the performance of Traverse $(L=1)$ drops as well. The reason is the length of FPs increase with a sharp trend of inclining so that much more merging operations are involved in Traverse, which is a non-trival overhead. 


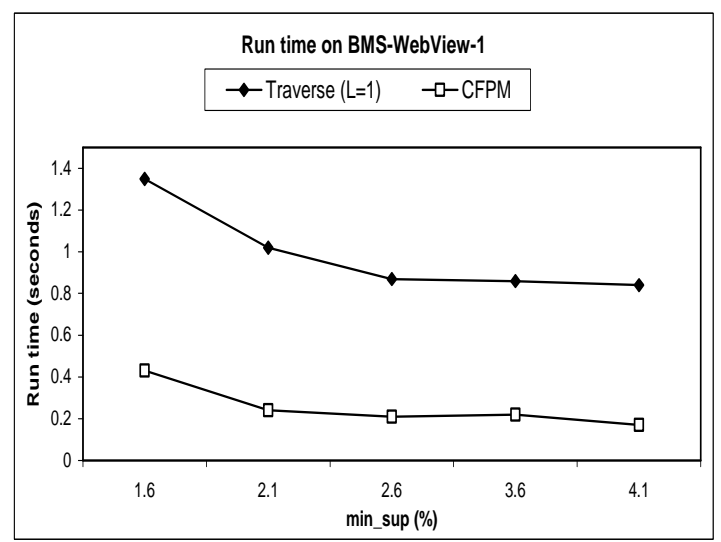

(a)

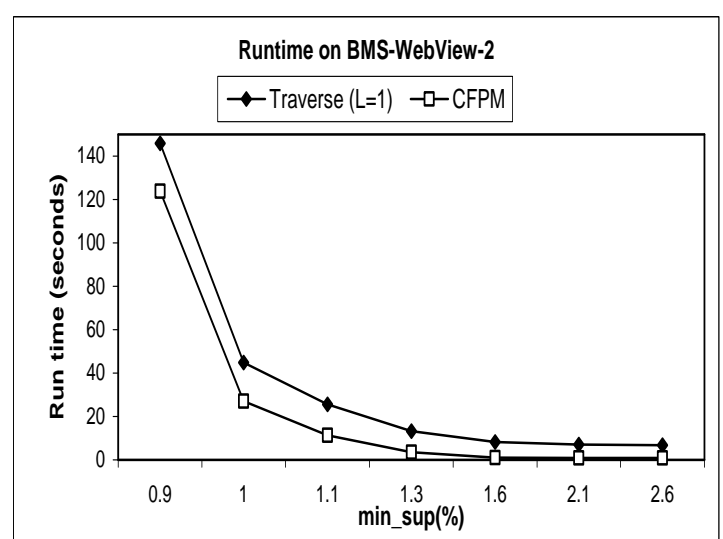

(b)

Figure 14: Performance of Traverse $(L=1)$ and CFPM

\begin{tabular}{c|c|c|c}
\hline \multicolumn{2}{c|}{ BMS-WebView-1 } & \multicolumn{2}{c}{ BMS-WebView-2 } \\
\hline min_sup $(\%)$ & Improved $(\%)$ & min_sup $(\%)$ & Improved $(\%)$ \\
\hline 2.1 & 313 & 0.9 & 118 \\
2.6 & 425 & 1.0 & 166 \\
3.6 & 414 & 1.1 & 226 \\
4.1 & 390 & 1.3 & 371 \\
4.6 & 494 & 1.6 & 783 \\
\hline
\end{tabular}

Table 3: Improvement of CFPM over Traverse $(L=1)$

Inspiringly, this problem has been fi xed by CFPM. We use candidate-generation-and-test approach to mine short FPs and use Traverse to mine long FPs. We use $L=3$ for our experiments. The improvements is very good. The comparison of experimental results of using Traverse to mine all FPs and use CFPM are shown in Figure 14. The improved percentages are shown in Table 3. From Table 3 we can see at minimum support threshold $2.1 \%$, the run time of CFPM is about 3 times faster than that of using Traverse $(L=1)$. At minimum support threshold $0.9 \%$, the run time of CFPM is about $118 \%$ faster than that of using Traverse $(L=1)$. The reasons are as follows: (1) As most of transactions of web logs are relatively short, most of FPs are mined before mining on the IFP-tree. (2) Use the minimum length of FPs, many transactions are already pruned before building the IFP-tree, so that the search space is decreased.

We also notice that after the first scan on the database we accumulate the frequency for all 
items to prune infrequent items before building an IFP-tree. At the point, in fact we already get all the 1-item FPs. In addition, we can also get all the 2-item FPs, ..., until $L$-item FPs at the building IFP-tree phase. From experiments we have conducted, we fi nd this usually take only about $0.1 \%$ of the total run time of building an IFP-tree. Furthermore, as we already get the frequent patterns with length less than or equal to a predefi ned minimum FP length $L$, we prune all the transactions with less than or equal to $L$ frequent items. This dramatically reduce the size of IFP-tree, as a big percentage of web log transactions are short transactions less than or equal to $L$. Experiments show these measures can dramatically improve the effi ciency of both building phase and mining phase.

\section{Conclusions and Discussions}

We have studied the problem of mining frequent patterns (FPs). We have done extensive experiments to examine the features of web logs and the characteristics of FPs presented in such data, in comparison to artifi cial supermarket transaction data and relational data. We also have conducted experiments to compare the performance of candidate-generationand-test approach and the pattern-growth approach, which are two main streams in frequent pattern mining fi eld. Our experiments show that neither the candidate-generationand-test approach nor the pattern-growth approach can cater for web logs. In particular, the candidate-generation-and-test approach outperforms the pattern-growth approach on mining short patterns, while pattern-growth approch is better on mining long patterns.

We have proposed improvements to current frequent mining approaches for better effi ciency of frquent pattern mining on web logs. We propose our new algorithm - Combined Frequent Pattern Mining (CFPM), which use a pre-defi ned minimum length $(L)$ of FPs. In CFPM, short patterns with length less than or equal to $L$ are mined by candidategeneration-and-test approach and patterns longer than $L$ are mined by pattern-growth approach. Experimental results show that CFPM can signifi cantly improve the performance of pattern-growth approach by $1.2 \sim 7.8$ times on frequent pattern mining on web log data.

\section{References}

[1] R. Agrawal and R. Srikant. Fast algorithms for mining association rules. In Proc. 1994 Int'l Conf. on Very Large Data Bases, pages 487-499, 1994. 
[2] R. Cooley, B. Mobasher and J. Srivastava. Web mining: Information and pattern discovery on the world wide web. In Proc. of the Int'l Conf. on Tools with Artificial Intelligence, IEEE, Newport Beach, pages 558-567, 1997.

[3] J. Han, J. Pei and Y. Yin. Mining frequent patterns without candidate generation. In Proc. of 2000 ACM-SIGMOD Conf. on Management of Data (SIGMOD-2000), Dallas TX, USA, 2000.

[4] G. Liu, H. Lu, Y. Xu and J. X. Yu. Ascending frequency order prefi X-tree: effi cient mining of frquent patterns. In Proc. of DASFAA 2003E, 2003.

[5] J. Pei, J. Han, B. Mortazavi-asl and H. Zhu. Mining access patterns effi ciently from Web logs. In Proc. of PAKDD-2000, 2000.

[6] Y. Xiao and M. H. Dunham. Effi cient mining of traversal patterns. In Data and Knowledge Engineering, 39(2), pages 191-214, 2000.

[7] J. Srivastava, R. Cooley, P. Deshpande, and P. Tan. Web usage mining: discovery and application of usage patterns from Web data. In SIGKDD Explorations, 2000.

[8] S. Dua, E. Cho, and S. Iyengar. Discovery of frequent patterns and user characteristics from Web access logs: a framework for dynamic Web personalization. In 3rd IEEE Symposium on Application-specific Systems and Software Engineering Technology (ASSET'00), 2000.

[9] R. Kosala and H.Blockeel. Web mining research: a survey. In ACM SIGKDD Explorations, 2000.

[10] M. H. Dunham. Data mining introductory and advanced topics, Pearson education, Inc 2003.

[11] R. Srikant and R. Agrawal. Mining quantitative association rules in large relational tables. In ACM SIGMOD International Conference on Management of Data, 1996.

[12] M.S. Chen, J. Han and P.S. Yu. Data Mining: An Overview from a Database Perspective. In IEEE Transactions on Knowledge and Data Engineering 8(6), pages 866-883, 1996.

[13] R. Agrawal, C. Aggarwal and V.V.V. Prasad. A tree projection algorithm for generation of frequent itemsets. In Journal of Parallel and Distributed Computing (Special Issue on High Performance Data Mining, 2000.

[14] S. Brin. Extracting patterns and relations from the World Wide Web. In WebDB Workshop at 6th International Conference on Extending Database Technology, EDBT'98, 1998. 
[15] C. Choo, B. Detlor and D. Turnbull. Information Seeking on the Web - An Integrated Model of Browsing and Searching. In ASIS Annual Meeting, 1999.

[16] R. Cooley, B. Mobasher and J. Srivastava. Grouping web page references into transactions for mining world wide web browsing patterns. In Technical Report TR 97-021, University of Minnesota, Dept. of Computer Science, Minneapolis, 1997.

[17] P. Pirolli, J. Pitkow and R. Rao. Silk from a sow's ear: Extracting usable structures from the web. In Conference on Human Factors in Computing Systems (CHI-96), Vancouver, British Columbia, Canada, 1996.

[18] R. J. Bayardo. Effi ciently mining long patterns from databases. In Proc. of SIGMOD’98, pages 85-93, 1998.

[19] P. Tan and V. Kumar. Mining Association Patterns in Web Usage Data. In International Conference on Advances in Infrastructure for e-Business, e-Education, eScience, and e-Medicine on the Internet, 2002.

[20] E. Keogh C. Blake and C.J. Merz. UCI repository of machine learning databases. http://www.ics.uci.edu/ mlearn/MLRepository.html, 1998.

[21] R. Agrawal and R. Srikant. Mining sequential patterns. In Proc. 1995 Int. Conf. Data Engineering, pages 3-14, Taipei, Taiwan, March 1995. 